\title{
ARTUS: The Tune Measurement System at RHIC*
}

\author{
A. Drees, M.Brennan, R. Connolly, R. Michnoff, J. DeLong \\ Collider-Accelerator Department, Brookhaven National Laboratory, Upton NY 11973
}

\begin{abstract}
The super-conducting Relativistic Heavy Ion Collider (RHIC) with two separate rings and six combined interaction regions will provide collisions between equal and unequal heavy ion species up to $\mathrm{Au}$ ions in typically 60 bunches. The betatron tunes of the two beams are among the most important parameters to be measured. The tunes have to be acquired at any moment during accelerator operation and in particular during the acceleration process. At RHIC the tune measurement device (ARTUS) consists of a fast horizontal and vertical kicker magnet and a dedicated beam position monitor in each ring. The system laycut is described and first experiences from operation is reported.
\end{abstract}

\section{INTRODUCTION}

This spring, 2000, RHIC is still being commissioned. Single bunched beam is injected successfully into one of the two rings ${ }^{1}$ (blue) and circulated with lifetimes of up to an hour. No attempts have been made to accelerate yet. Thus the fractional tunes could be measured at injection using the large injection oscillations in both planes and with circulating beam at injection energies. To do the latter, betatron oscillations are excited with a fast transverse kicker magnet [1] and transverse beam positions are measured on subsequent turns with one dedicated dual plane Beam Position Monitor (BPM) [2]. The fractional tunes are extracted from the position data by performing a FFT analysis. During the earlier commissioning run last year, in summer 1999, ARTUS² [3] did not include a specific BPM to be used for tune measurements only. Instead, any BPM from a total of 246 per ring could be selected. The kicker, setup to kick only once at a time, was triggered by using a modified BPM readout electronic board [4]. While it has certain advantages to have a choice at what location the beam position data is taken the system lacked kicking strength especially for higher beam energies and flexibility in triggering and control. Additionally, problems emerged from a competition situation between the RHIC orbit system on one side and the tune measurements on the other. Accordingly

1) the two rings are named "blue" and "yellow"

2) A Rhic TUne measurement System

*Work performed under the auspices of the U.S. Department of Energy. 
the ARTUS system layout was changed during the last year. Now a movable BPM is assigned to the tune meter, featuring a different trigger system for both, kicker and BPM. The capability of multiple kicks (turn-by-turn) was added to ensure a decent signal amplitude at all beam energy settings. The new readout electronic developed for the movable BPM and the control system is now installed in its own VME crate.

\section{KICKER AND BPM}

Each ring has two kicker modules with four $2 \mathrm{~m}$-long stainless steel striplines mounted on ceramic stand-offs spaced $1 \mathrm{~m}$ apart allowing both horizontal and vertical kicks. The two kickers are connected in series to provide $4 \mathrm{~m}$ of stripline kickers. Each stripline subtends an angle of $70^{\circ}$ at an aperture of $7 \mathrm{~cm}$. The assembly is designed to give $50 \Omega$ impedance when opposing lines are driven in the difference mode. Figurel shows one kicker module in the assembly area.

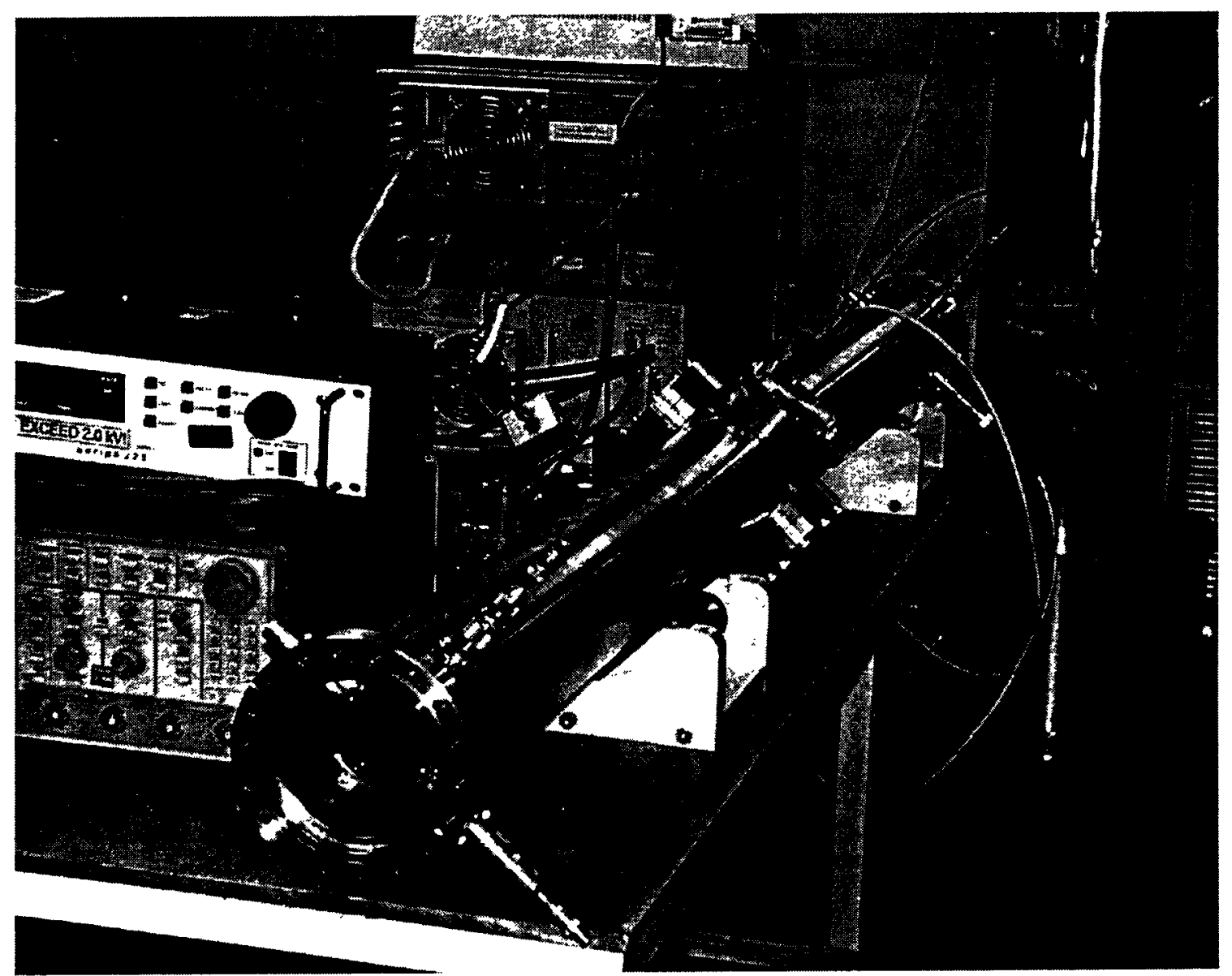

FIGURE 1. One kicker module on a work bench in the assembly area. 
Each of the four planes can be powered independently. Only pulsed power is used for early operation of RHIC. The kick pulses are generated by fast FET switches [5] producing a $140 \mathrm{~ns}$ long pulse. By centering this pulse on the measured bunch single bunch excitation is possible with even up to 120 bunches per ring where the bunch spacing is about $110 \mathrm{~ns}$. All switches for all striplines in both rings are charged by one $5 \mathrm{kV} / 2 \mathrm{~A}$ power supply. The kick angle after one pulse with $3 \mathrm{kV}$ received by an ion going through the kickers is approximately $10 \mu \mathrm{rad}$ at injection energy $(\gamma \approx 10)[6]$. The effect of such a kick translates into a beam offset given by:

$$
\delta_{x, y}=\Theta \sqrt{\beta_{x, y}\left(s_{0}\right) \beta_{x, y}(s)} \sin \psi_{x, y}(s),
$$

where $\beta_{x, y}\left(s_{0}\right)$ is the $\beta$-function at the location $s_{0}$ of the kicker and $\beta_{x, y}(s)$ are the respective horizontal or vertical $\beta$-function and phase advance at location $s$. Table 1 summarizes the values of the $\beta$ functions at the location of the kickers and BPMs respectively for the lattice used for injection and storage in the spring 2000 run $\left(\beta^{*}\right.$ values of $8 / 3 / 8 / 3 / 3 / 3 \mathrm{~m}$ and $1 / 3 / 8 / 3 / 3 / 3 \mathrm{~m}$ at the 6 interactin points respectively). The design values for injection energy are added for comparison. The asymmetry in the lattice function for the spring 2000 run originates from a number of missing power supplies which would be needed to create the design lattice. The actual lattice strongly favors the horizontal plane when resulting beam offsets are calculated.

TABLE 1. $\beta$ functions at the location of the blue kicker and movable BPM for the run 2000 lattices. The values for the yellow ring are analogous.

\begin{tabular}{|l|c|c|c|c|c|c|c|}
\hline device & $\mathrm{s}(\mathrm{m})$ & \multicolumn{2}{|c|}{ inj. 2000} & \multicolumn{2}{c|}{ store 2000 } & \multicolumn{2}{c|}{ inj. design } \\
& & $\beta_{x}(\mathrm{~m})$ & $\beta_{y}(\mathrm{~m})$ & $\beta_{x}(\mathrm{~m})$ & $\beta_{y}(\mathrm{~m})$ & $\beta_{x}(\mathrm{~m})$ & $\beta_{y}(\mathrm{~m})$ \\
\hline kicker & 2491.7 & 81.3 & 5.6 & 82.9 & 5.5 & 33.0 & 15.5 \\
BPM & 2503.1 & 189.0 & 33.8 & 192.4 & 33.5 & 66.0 & 13.0 \\
\hline
\end{tabular}

The movable BPM used for the tune meter is realized by short circuited transmission lines of $23 \mathrm{~cm}$ length, with a design impedance of $50 \Omega$, and an aperture of 7 $\mathrm{cm}$. They are movable in both transverse directions by about $+/-2 \mathrm{~cm}$. This feature is used to guarantee a well centered beam between the two striplines. The BPM is located in the warm straight section between the Q3 and Q4 magnets. This BPM can be converted into a resonant device with a high Q-value and a significantly improved resolution. The $\beta$ functions are listed in table 1 .

\section{TRIGGER AND DATA ACQUISITION}

Figure 2 sketches the signal processing and triggering of both, the BPMs and the kickers. 


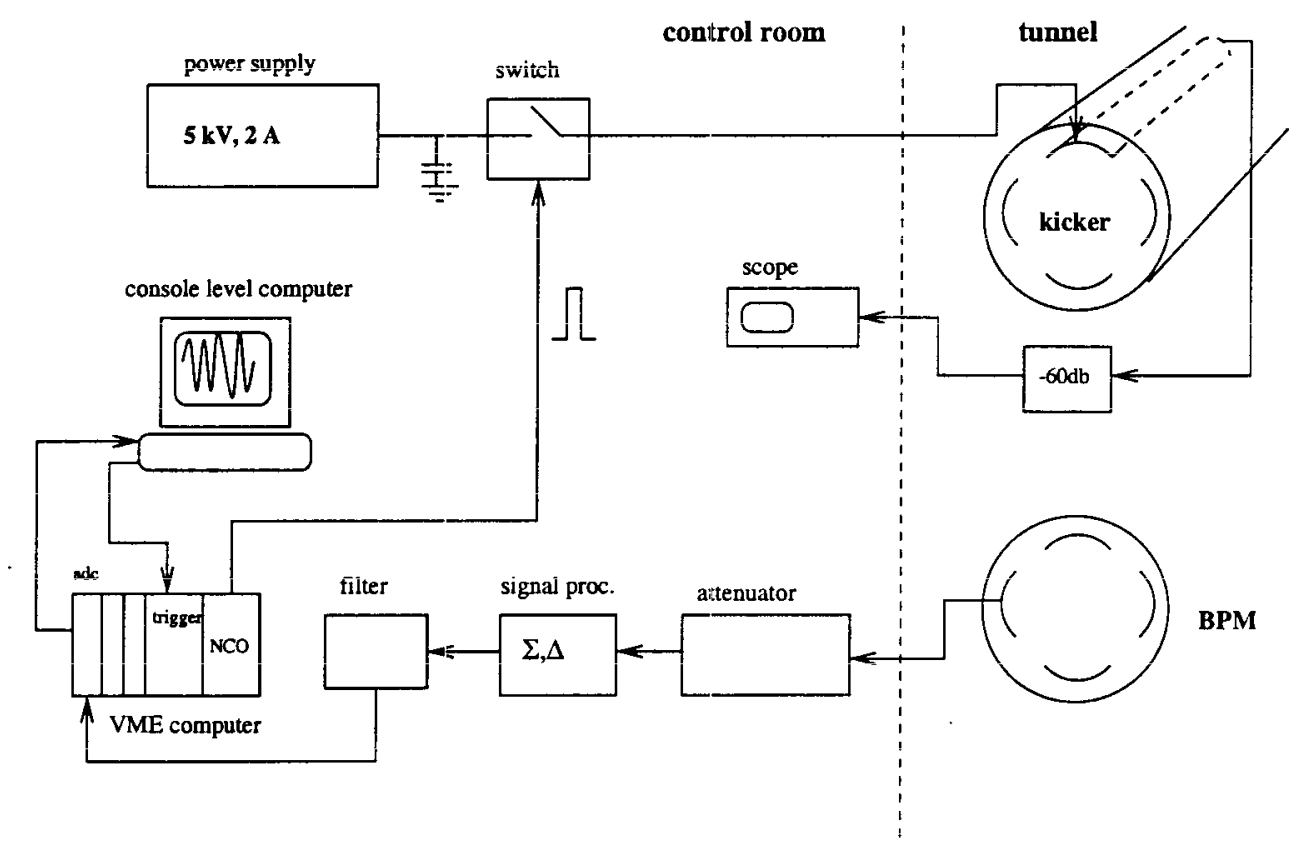

FIGURE 2. Block diagram of kicker and BPM readout. For simplicity wiring for only one plane is drawn while all four BPM and kicker striplines are connected in the tunnel.

The FET switches are triggered by a TTL pulse of $200 \mathrm{~ns}$ width from a $\mathrm{Nu}-$ merically Controlled Oscillator (NCO) board [7]. The NCO outputs pulses with a certain phase and a frequency of up to $20 \mathrm{MHz}$. The resolution is $0.09^{\circ}$ and 11.6 $\mathrm{mHz}$ respectively. The phase and the frequency are parameters which can be set remotely from a console level computer. A total of $4 \mathrm{NCO}$ channels is available for the two planes per ring. One NCO channel is connected to both striplines in one plane. Thus the two striplines in each plane are fired in accordance with the set frequency resulting in kicks in both directions. Figure 3 shows the kick pattern induced by the $\mathrm{NCO}$ on two striplines in one plane of the kicker in the blue ring. By selecting a NCO frequency close to the horizontal and vertical betatron frequency the beam is kicked resonantly enhancing the effect on the beam significantly if compared with a single kick. The enhancement factor was estimated to be of the order of 10 for a limited number of kick pulses. However, a set point equal or very close to the betatron frequency was shown to kick the beam out of the ring if the number of turns was too high. In order to control the total number of kicks, the $\mathrm{NCO}$ is triggered synchronously with the beam by using a V124 board [8] allowing the number of beam synchronous triggers to be set to any desired value up to 65535. Differences in the kicker efficiency in the two planes could successfully be compensated for by varying the number of kicks for the two NCO channels.

The V124 module receives and decodes the beam synchronous event link [9]. The raw data acquisition frorn the two BPM planes is triggered by other channels of the same V124 board where a total of 8 channels is available. Each channel 


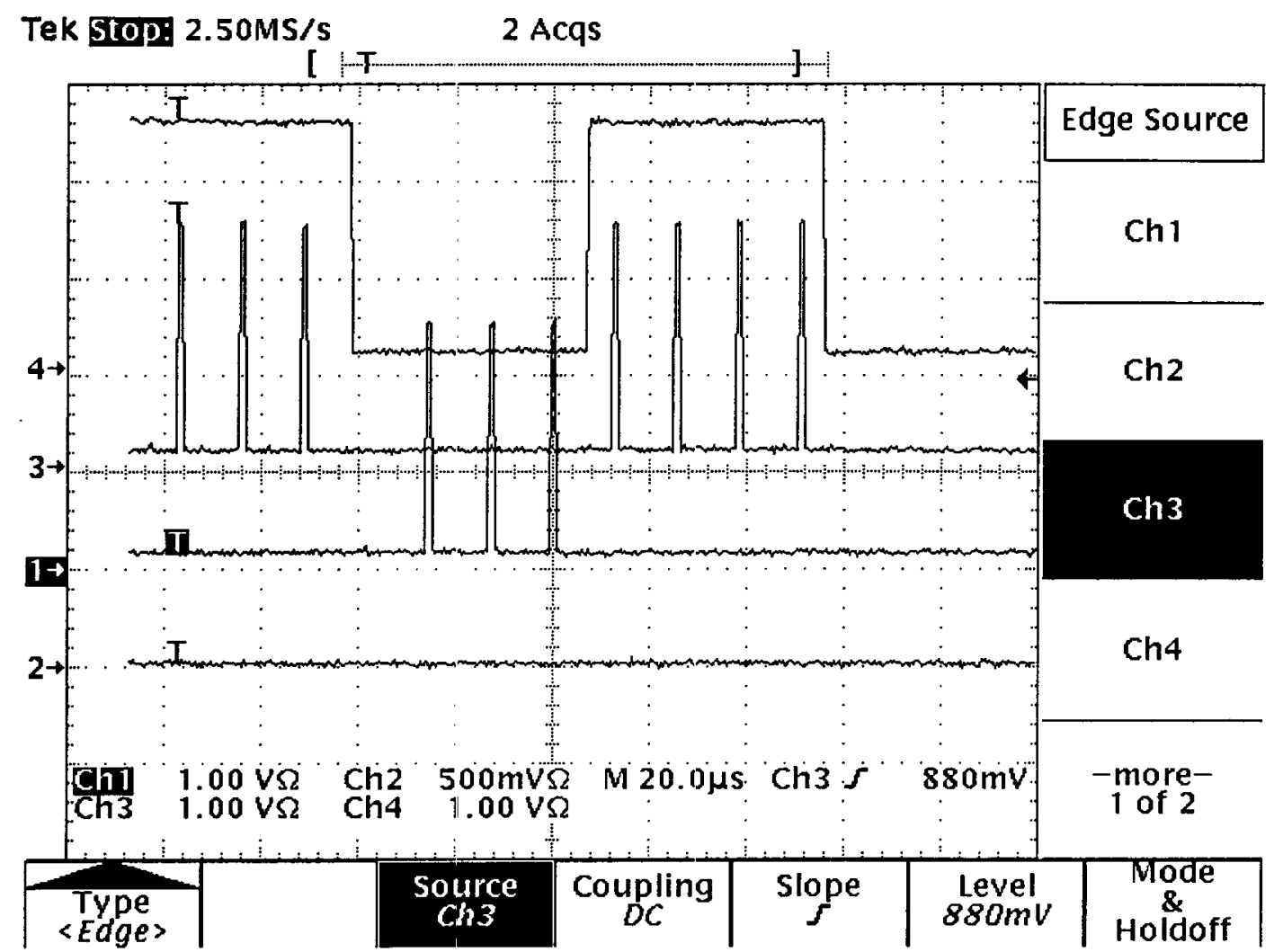

FIGURE 3. Kick pattern from $\mathrm{NCO}$ at a frequency of $10.3 \mathrm{kHz}$. Signals from top to bottom: 1) NCO input frequency of $10.3 \mathrm{kHz}, 2$ ) resulting kick pattern from inside stripline, 3) resulting kick pattern from outside stripline.

for BPM readout and the kicker trigger has the appropriate delay so, on turnby-turn acquisition, the same bunch will be kicked and observed on the BPM. Start turn number, total number of turns for acquisition and time delays are all parameters which can be set remotely from a console level computer. The V124 allows the tune measurement to be triggered by any event broadcasted on the beam synchronous link such as a 4-sec-event, 1-Hz-event, injection-event, startacceleration-event or on demand. Thus the tunes can be easily correlated any time with other instrumentation and the status of the blue and yellow ring respectively.

The raw (bipolar) signal frcm the movable BPM is attenuated by a programmable attenuator of 0-30 db. Then two signal processing NIM modules compute the two difference signals and the surn of all stripline signals for each ring. Its mono-polar output signal is filtered by two programmable Krohn-Hite filters model 3995 and digitized by a Hytec VME adc module [10]. The result is then output for the data analysis. 


\section{DATA ANALYSIS AND FIRST EXPERIENCE}

The data is analyzed by performing a fast Fourier Transformation (FFT) on the position data for $\mathrm{N}$ turns. To comply with the FFT requirements $\mathrm{N}$ should be set to any power of 2. This is typically done using an application on a console level computer and passed through to the V124 trigger module. In order to make the tune measurements available to any possible client, data analysis including the FFT is done on the front end computer. Application software is used only for displaying purposes and to control the programmable data acquisition modules. The application software includes the following features:

- One window per plane is displaying the raw data as well as the FFT spectrum including the results from a peak finding algorithm and Gauss-fit.

- Mountain-range plots from up to 25 consecutive FFT spectra allow tracking of tune changes during periods of fast change in the RHIC rings. Figure 4 shows a typical mountain-range plot from simulated data.

- Upper and lower limits for the peak finding algorithm can be set to avoid ambiguous results due to coupling between the two planes.

- The data set used for the FFT can be selected off-line after visual inspection to guarantee the quality of the data points used in the measurement.

- Raw data can be archived for all four planes and each measurement for later analysis.

For $\mathrm{N}<64$ no reliable tune measurement can be performed. While a set of $\mathrm{N}=1024$ turns would result in a measurement accuracy of $\delta q=1 \cdot 10^{-3}, \mathrm{~N}<256$ turns give poor results and accuracies of down to some $10^{-2}$. Although practically useless for dynamic tune measurements, for instance during an energy ramp, even poor accuracy data turned out to be very useful during the first days of commissioning. With such a bad resolution the (very large negative) horizontal chromaticity could still be measured successfully during the first days using ARTUS. Figure 5 shows a typical display of a tune measurement during a radial scan. For this measurement, the betatron oscillation was excited at injection energy using 10 consecutive kicks 10 turns after the start of data acquisition. The dotted line in the upper graph indicates the start of the kicks. Only 128 data points following the start of the kicks have been selected for the FFT to give a clear tune signal.

\section{CONCLUSION}

At the moment ARTUS has been used successfully in the blue ring and at injection energies. Tune scans, chromaticity and coupling measurements were performed using it. Since no successful attempts to accelerate have been made so far, the system could not be tuned for dynamic measurements or at storage energies yet. 


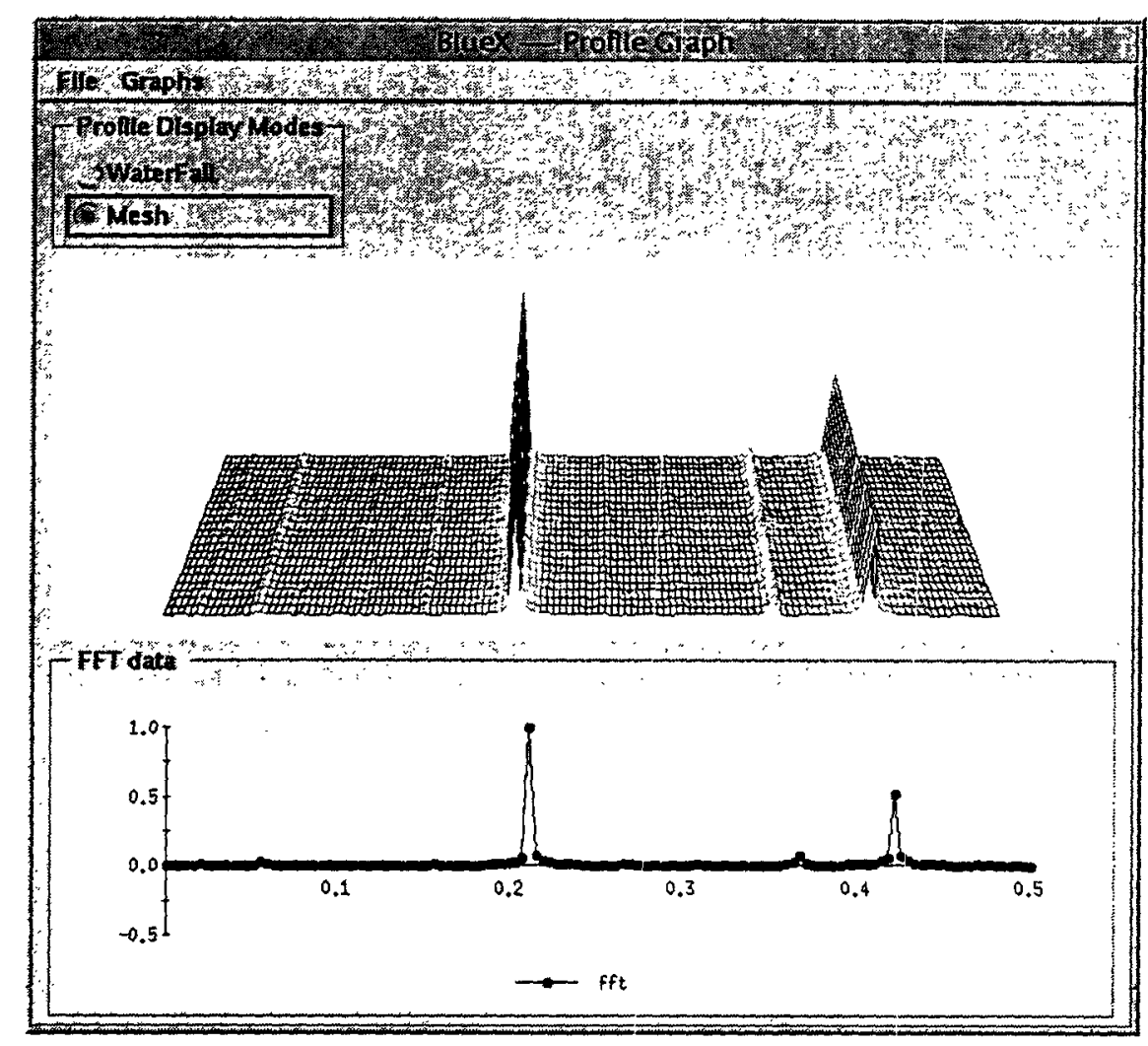

FIGURE 4. Typical mountain-range display from ARTUS application, taken with simulated data.

Although the beta function at the location of the kicker as well as at the location of the BPM favor the horizontal plane a decent signal could be created in the vertical plane by carefully setting the kicking frequency and increasing the number of kicks. Because of the flexible trigger system and the programmable NCO the system is capable of measuring the tunes at higher energies and during the ramp at a rate of up to $1 \mathrm{~Hz}$. However, increasing the number of kicks is shown to cause significant beam losses. Therefore, a more sensitive BPM would be desirable for the future.

\section{REFERENCES}

1. J. Xu et al., "The Transverse Damper System for RHIC", Proceedings of the Particle Accelerator Conference (PAC) in San Francisco, 1991.

2. P. Cameron et al., "RHIC Beam Position Monitor Assemblies", IEEE Proc., 1995 PAC. 

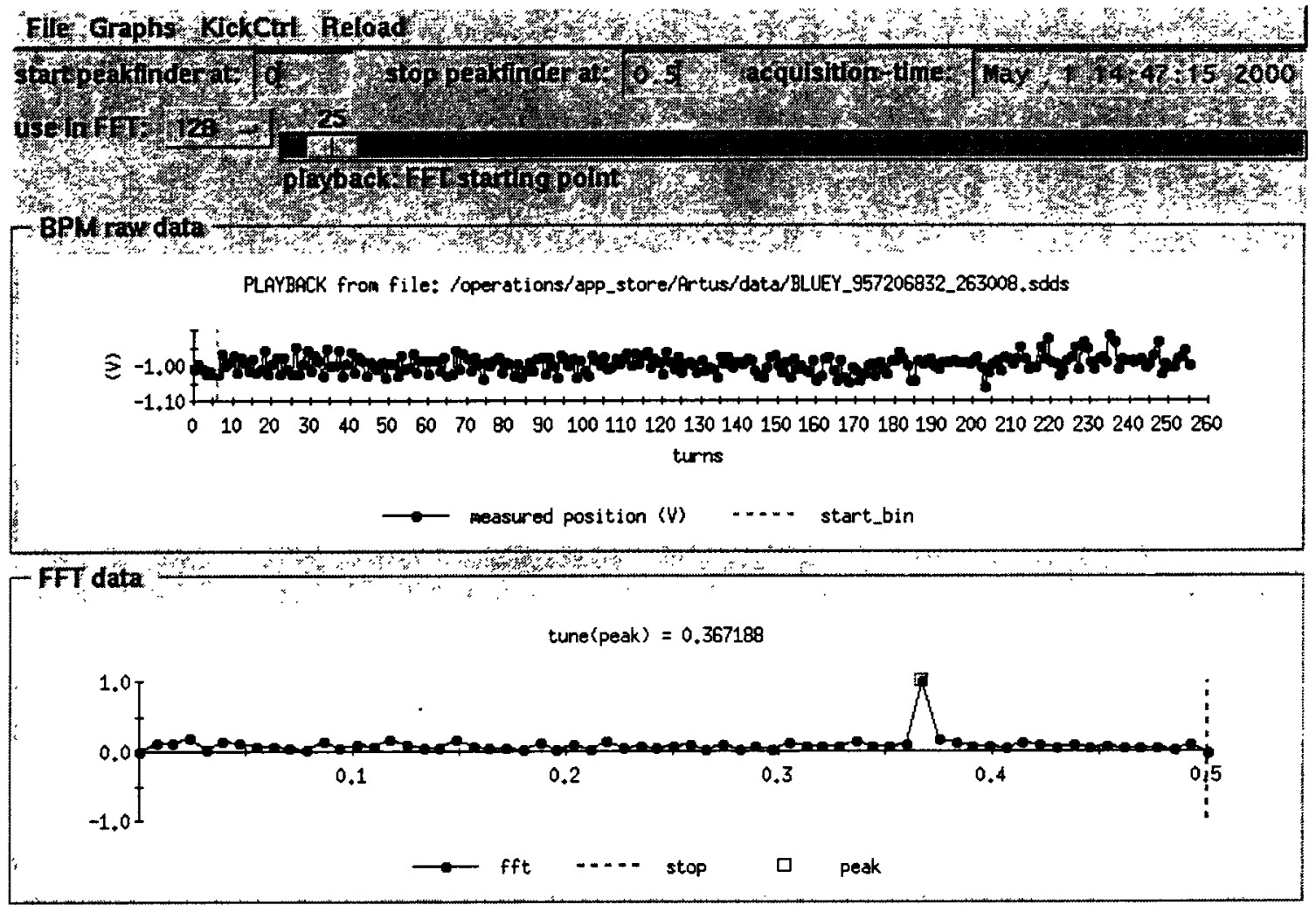

FIGURE 5. Typical display from ARTUS application for one tune measurement in the vertical plane in the blue ring.

3. P. Cameron, R. Connolly, J. Cupolo, A. Drees, W. Ryan, T. Shea, R. Sikora, D. Trbojevic, N. Tsoupas, "The RHIC Tune Measurement System",Proc. of PAC 1999, New York (p2117).

4. W. Ryan, T. Shea, P. Cerniglia, C. Degen, "A Prototype BPM Electronics Module for RHIC", Proc. PAC 1993 (p. 2310).

5. Behlke Electronic GmbH, http://www.euretek.com/

6. P. Cameron, R. Connolly, A. Drees, W. Ryan, H. Schmickler, T. Shea, D. Trbojevic, "ARTUS: A Rhic TUne Measurement System", RHIC/AP/98-125, internal note.

7. J. DeLong, private communication.

8. H. Hartmann, T. Kerner, "RHIC beam synchronous trigger module", Proc. PAC 1999 (p. 696).

9. T. Kerner, C. R. Conkling Jr., B. Oerter, "V123 Beam Synchronous Encoder Module", Proc. PAC 1999 (p. 699).

10. Hytec Electronics Ltd.,http://www.hytec-electronics.co.uk 